University of Nebraska - Lincoln

DigitalCommons@University of Nebraska - Lincoln

$2-1-2001$

\title{
Effects of external electric field on the interfacial properties of weakly dipolar fluid
}

\author{
V.B. Warshavsky \\ University of Nebraska-Lincoln \\ T.V. Bykov \\ University of Nebraska-Lincoln \\ Xiao Cheng Zeng \\ University of Nebraska-Lincoln, xzeng1@unl.edu
}

Follow this and additional works at: https://digitalcommons.unl.edu/chemzeng

Part of the Chemistry Commons

Warshavsky, V.B.; Bykov, T.V.; and Zeng, Xiao Cheng, "Effects of external electric field on the interfacial properties of weakly dipolar fluid" (2001). Xiao Cheng Zeng Publications. 48.

https://digitalcommons.unl.edu/chemzeng/48

This Article is brought to you for free and open access by the Published Research - Department of Chemistry at DigitalCommons@University of Nebraska - Lincoln. It has been accepted for inclusion in Xiao Cheng Zeng Publications by an authorized administrator of DigitalCommons@University of Nebraska - Lincoln. 


\title{
Effects of external electric field on the interfacial properties of weakly dipolar fluid
}

\author{
V. B. Warshavsky, T. V. Bykov, and X. C. Zeng \\ Department of Chemistry, University of Nebraska-Lincoln, Lincoln, Nebraska 68588
}

(Received 14 July 2000; accepted 6 October 2000)

\begin{abstract}
In the framework of modified mean-field density-functional theory, effects of a uniform electric field on the interfacial properties of a model dipolar fluid [Teixeira and Telo da Gama, J. Phys.: Condens. Matter 3, 111 (1991)] are studied. Both density and orientational order-parameter profiles of the planar vapor-liquid interface are obtained as a function of the field strength. For the dipolar fluids with reduced dipole moment $\mu_{0}^{*} \leqslant 1$, we find that the field (under the condition $\mu_{0}^{*} E^{*} / T^{*} \ll 1$ ) can shift the surface tension by few percent. We also find that the electric field actually reduces the thermodynamical surface tension but enhances the mechanical surface tension at the equimolar dividing surface. To detect these field effects on the surface tension we estimate the field strength which can be as high as $10^{8} \mathrm{~V} / \mathrm{m}$. (C) 2001 American Institute of Physics.
\end{abstract}

[DOI: 10.1063/1.1329345]

\section{INTRODUCTION}

Understanding the effects of an applied electric field on the interfacial properties of polar fluids is fundamentally important not only in its own right but also in the study of vapor-liquid nucleation in the presence of an electric field. Examples include ion-induced nucleation ${ }^{1-10}$ and nucleation in a uniform electric field. ${ }^{11-16}$ The classical theory of nucleation predicts that the barrier height to nucleation is proportional to the cube of the surface tension in the absence of electric field. ${ }^{13}$ Consequently, if the applied field can cause a small shift in the surface tension it may lead to an appreciable change in the rate of nucleation according to the classical theory. This is because the rate depends exponentially on the barrier height.

Interfacial behavior of a model dipolar fluid (similar to the Stockmayer fluid) in the absence of the external field has been thoroughly studied by Teixeira and Telo da Gama ${ }^{17,18}$ using the modified mean-field (MMF) density-functional theory. In that theory, an expansion of the grand canonical potential in powers of the anisotropic (multipolar) part of the intermolecular pair potential was used. Teixeira and Telo da Gama showed that larger molecular dipole moments generally yield a higher surface tension. They also showed that an interfacial ordering can be induced solely by the multipolar forces. Using the MMF density-functional theory Talanquer and Oxtoby ${ }^{19}$ studied the vapor-liquid nucleation of the Stockmayer fluid. To investigate the interfacial properties of strongly dipolar fluids, Frodl and Dietrich ${ }^{20,21}$ developed a more sophisticated density-functional approach without using the expansion of the grand canonical potential. They showed that when the reduced dipole moment is less than 1 (weakly dipolar), the MMF theory ${ }^{17}$ gives reasonably good results compared to their theory (e.g., the discrepancy in the critical temperature is less than $10 \%$ ). The interfacial structures of a dipolar fluid was also studied by Forstmann and co-workers $^{22,23}$ using a weighted density-functional theory and integral equation theory.
When a dipolar fluid is in the presence of an external electric field, both of its bulk and interfacial properties will differ from those in the absence of the field. In earlier theoretical studies ${ }^{24-27}$ macroscopic theories based on thermodynamics were developed and the thermodynamic equations for the nonuniform system in the external electric field were obtained. Both the normal electric field ${ }^{25,26}$ and an arbitrarily oriented electric field ${ }^{24,27}$ to the interface were considered.

Several works based on molecular approaches have recently been published. Using the weighted density-functional theory Patra and Ghosh ${ }^{28}$ and Das et al. ${ }^{29}$ studied structural properties of dipolar liquids near charged solid surfaces which generate a nonuniform external electric field. Computer simulations were also employed by many researchers to investigate the effects of external field on various properties of dipolar fluids, e.g., the saturation of dielectric constant, $^{30}$ electrostriction, ${ }^{31}$ and coexistence phase behavior. ${ }^{32-34}$ Kiyohara et al. ${ }^{34}$ examined the influence of the dielectric constant of surrounding media on the vaporliquid phase coexistence of Stockmayer fluid in an external electric field. Woodward and Nordholm ${ }^{35}$ devised a densityfunctional approach to study the phase behavior of dipolar hard-sphere fluid in an electric field. They used the so-called independent pair approximation free-energy functional in which orientational correlations between dipoles were accounted for via the constrained reference state averaged Mayer function. With this free-energy functional they explored how the shape of the system affects the phase coexistence in the electric field. Groh and Dietrich ${ }^{36}$ studied the effects of external magnetic fields on the isotropic and ordered magnetic phase coexistence in an ellipsoidal shaped system.

Thus far, most theoretical or computational studies have only concerned with the bulk properties and phase coexistence of dipolar fluids in external fields. The properties of vapor-liquid interfaces of dipolar fluids in applied electric fields remain unexplored from either molecular-based theory 
or computer simulation. The present work is an effort toward this direction. The paper is organized as a follows. In Sec. II the extension of the MMF density-functional theory for the weakly dipolar fluid in a uniform electric field is developed. In Sec. III the influence of the electric field on the bulk properties and phase coexistence is presented. Results of density and orientational order-parameter profiles as well as the surface tension in the electric field are presented in Sec. IV. A discussion is given in Sec. V.

\section{DENSITY FUNCTIONAL THEORY OF DIPOLAR FLUID}

We consider a single-component dipolar fluid in the framework of density-functional theory. Let $\rho(\mathbf{r}, \omega)$ denote the number density of molecules at position $\mathbf{r}=(x, y, z)$ and orientation $\omega=(\theta, \phi)$ with respect to the space fixed coordinate. The number density can be written as

$$
\rho(\mathbf{r}, \omega)=\rho(\mathbf{r}) f(\mathbf{r}, \omega),
$$

where $\rho(\mathbf{r})$ is the total number density of molecules without specifying the orientation and $f(\mathbf{r}, \omega)$ is the orientational distribution function. The intermolecular interaction of the fluid is characterized by a pairwise intermolecular potential $\phi\left(\mathbf{r}_{1}, \mathbf{r}_{2}, \omega_{1}, \omega_{2}\right)$, where $\mathbf{r}_{1}$ and $\mathbf{r}_{2}$ denote positions of the two molecules and $\omega_{1}$ and $\omega_{2}$ are the orientations of molecular dipoles. For weakly dipolar fluids, we employed the perturbation approach to divide the intermolecular potential into two parts: a strong repulsive interaction $\phi_{\text {ref }}\left(\mathbf{r}_{1}, \mathbf{r}_{2}, \omega_{1}, \omega_{2}\right)$ and a relatively small perturbative attraction $\phi_{p}\left(\mathbf{r}_{1}, \mathbf{r}_{2}, \omega_{1}, \omega_{2}\right)=\phi\left(\mathbf{r}_{1}, \mathbf{r}_{2}, \omega_{1}, \omega_{2}\right)-\phi_{\text {ref }}\left(\mathbf{r}_{1}, \mathbf{r}_{2}, \omega_{1}, \omega_{2}\right)$. The former is treated as a reference system. Here, the reference and perturbative potentials of the model dipolar fluid are chosen in the same fashion as in Ref. 17, i.e.,

$$
\phi_{\text {ref }}(r)=\left\{\begin{array}{cc}
+\infty & r \leqslant d \\
0 & r>d
\end{array}\right.
$$

and

$$
\phi_{p}\left(r, \omega_{1}, \omega_{2}\right)= \begin{cases}0 & r \leqslant d \\ -4 \epsilon_{\mathrm{LJ}}(d / r)^{6}+\phi_{d d}\left(r, \omega_{1}, \omega_{2}\right) & r>d .\end{cases}
$$

In Eq. (2) $d$ is a hard-sphere diameter and $r$ is the intermolecular distance; in Eq. (3) $\epsilon_{\mathrm{LJ}}$ is the energy parameter of the Lennard-Jones potential and $\phi_{d d}$ is the interaction between two dipoles,

$$
\phi_{d d}=-\frac{3\left(\boldsymbol{\mu}_{1} \cdot \mathbf{r}\right)\left(\boldsymbol{\mu}_{2} \cdot \mathbf{r}\right)-\left(\boldsymbol{\mu}_{1} \cdot \boldsymbol{\mu}_{2}\right) r^{2}}{r^{5}},
$$

where $\boldsymbol{\mu}_{1}$ and $\boldsymbol{\mu}_{2}$ are the molecular dipole moments (with the same magnitude $\mu_{0}$ ). The dipole-dipole interaction can be conveniently written as a sum of spherical harmonics ${ }^{37}$

$$
\begin{aligned}
\phi_{d d}= & -\frac{\mu_{0}^{2}}{r^{3}} 4 \pi\left(\frac{8 \pi}{15}\right)^{1 / 2} \sum_{m_{1}, m_{2}, m_{3}} C\left(112 ; m_{1}, m_{2}, m_{3}\right) \\
& \times Y_{1 m_{1}}(\omega) Y_{1 m_{2}}\left(\omega^{\prime}\right) Y_{2 m_{3}}^{*}\left(\omega^{\prime \prime}\right),
\end{aligned}
$$

where $Y_{l m}$ are spherical harmonics and $C\left(112 ; m_{1}, m_{2}, m_{3}\right)$ are Clebsch-Gordan coefficients in the Rose convention; ${ }^{38}$ $\omega^{\prime \prime}$ sets the orientation of the intermolecular axis with respect to the space fixed coordinate system.

In practice, a certain approximation for the pair distribution function is needed to compute the free-energy functional. One simple approximation is the so-called randomphase approximation. As shown in many previous works, ${ }^{17-20}$ the random-phase approximation cannot provide a realistic description for the dipolar system. Teixeira and Telo da Gama $^{17}$ proposed a MMF approximation which is based on a low-density expression for the radial distribution function. With the MMF approximation, the grand canonical potential energy of the system in an external field $V_{\text {ext }}(\mathbf{r}, \omega)$ can be expressed as

$$
\begin{aligned}
\Omega[\rho(\mathbf{r}, \omega)]= & \int d \mathbf{r} f_{\mathrm{ref}} \rho(\mathbf{r}) \\
& -\int d \mathbf{r} d \omega \rho(\mathbf{r}) f(\mathbf{r}, \omega)\left(\mu-V_{\mathrm{ext}}(\mathbf{r}, \omega)\right) \\
& +\frac{k_{B} T}{2} \int d \mathbf{r} d \mathbf{r}^{\prime} d \omega d \omega^{\prime} \rho(\mathbf{r}) \rho\left(\mathbf{r}^{\prime}\right) \\
& \times f(\mathbf{r}, \omega) f\left(\mathbf{r}^{\prime}, \omega^{\prime}\right) e^{-\beta \phi_{\mathrm{ref}}\left(\mathbf{r}, \mathbf{r}^{\prime}\right)} \\
& \times\left(1-\exp \left[-\beta \phi_{p}\left(\mathbf{r}, \mathbf{r}^{\prime}, \omega, \omega^{\prime}\right)\right]\right)
\end{aligned}
$$

where $\mu$ is chemical potential, $T$ the temperature, $k_{B}$ is the Boltzman constant, and $\beta=1 / k_{B} T$. In Eq. (6), the Helmholtz free-energy density of the reference system has the form

$$
\begin{aligned}
f_{\text {ref }}(\rho(\mathbf{r}))= & f_{h}(\rho(\mathbf{r}))+k_{B} T \rho(\mathbf{r}) \\
& \times \int d \omega f(\mathbf{r}, \omega) \ln [4 \pi f(\mathbf{r}, \omega)],
\end{aligned}
$$

where the first term on the right-hand side is the free-energy density of the hard-sphere system, which is normally given in the Carnahan-Starling form, ${ }^{39}$ and the second term is due to the loss of the entropy caused by the orientational order of molecular dipoles. For isotropic molecular fluids, $f(\mathbf{r}, \omega)$ $=1 / 4 \pi$, and the second term becomes zero.

We consider the planar vapor-liquid interface in the $x-y$ plane with the external electric field along the $z$ axes. For the planar interface it is natural to choose the shape of the system as a slab. Thus, the density profile $\rho(z)$ depends only on the spatial variable $z$ and the orientation distribution function $f(z, \omega)$ no longer depends on the axial angle $\phi$ (in the $x-y$ plane). Moreover, $f(z, \omega)$ can be expressed as

$$
f(z, \omega)=\exp (\alpha(z, \omega)) / \int d \omega \exp (\alpha(z, \omega)),
$$

where $\alpha(z, \omega)$ is in terms of Legendre polynomials

$$
\alpha(z, \omega)=\alpha_{1}(z) P_{1}(\cos \theta)+\alpha_{2}(z) P_{2}(\cos \theta) .
$$

For weakly dipolar systems, for example, when the reduced dipole moment is less than 1, we follow Refs. 17 and 20 and consider only two terms in the expansion of $\alpha(z, \omega)$ with respect to Legendre polynomials. The two terms are needed at least because $\alpha_{1}$ is zero in the absence of the external 
field ${ }^{17-19,21}$ and in that case $\alpha_{2}$ will provide the leading contribution to the angular dependence of the density distribution function.

Using Eq. (5) and keeping only two terms in the expansion of the exponential function in Eq. (6) over the perturbative potential $\beta \phi_{p}$, we can obtain the following expression for the grand canonical potential per unit of area:

$$
\begin{aligned}
& \Omega\left[\rho(z), \eta_{1}(z), \eta_{2}(z)\right] / A \\
& =\int_{-\infty}^{\infty} d z\left\{f_{h}(\rho(z))+k_{B} T \rho(z)\right. \\
& \left.\quad \times \int d \omega f(z, \omega) \ln [4 \pi f(z, \omega)]\right\} \\
& \quad-\int_{-\infty}^{\infty} d z \mu \rho(z)-\int_{-\infty}^{\infty} d z \mu_{0} E \rho(z) \eta_{1}(z) \\
& \quad+\frac{1}{2} \int_{-\infty}^{\infty} \int_{-\infty}^{\infty} d z d z^{\prime} \phi_{\mathrm{eff}}\left(z, z^{\prime}\right) \rho(z) \rho\left(z^{\prime}\right),
\end{aligned}
$$

where $A$ is the area of the planar vapor-liquid interface and $E$ the magnitude of the uniform external electric field. In Eq. (10),

$$
\begin{aligned}
\phi_{\mathrm{eff}}\left(z, z^{\prime}\right)= & \phi_{0}(\bar{z})+\eta_{2}(z) \eta_{2}\left(z^{\prime}\right) \phi_{1}(\bar{z})+\left(\eta_{2}(z)\right. \\
& \left.+\eta_{2}\left(z^{\prime}\right)\right) \phi_{2}(\bar{z})+\eta_{1}(z) \eta_{1}\left(z^{\prime}\right) \phi_{3}(\bar{z}),
\end{aligned}
$$

where $\bar{z}=z-z^{\prime}$ and the exact expressions for the functions $\phi_{0}(z), \phi_{1}(z), \phi_{2}(z)$, and $\phi_{3}(z)$ are given in Refs. 17 and 18. Note that in Refs. 17 and 18 different notations are used, where $V$ is $-\phi_{1}, \phi_{2}$ is $-\phi_{2}$, and $\phi_{1}$ is $-\phi_{3}\left[\phi_{0}(z)\right.$ is the same]. The orientation order parameters $\eta_{1}(z)$ and $\eta_{2}(z)$ are basically the coefficients in the expansion of orientation distribution function $f(z, \omega)$ with respect to the Legendre polynomials

$$
\begin{aligned}
& \eta_{1}(z)=\int d \omega P_{1}(\cos \theta) f(z, \omega), \\
& \eta_{2}(z)=\int d \omega P_{2}(\cos \theta) f(z, \omega) .
\end{aligned}
$$

Applying the variational principle to the grand canonical potential [Eq. (10)] with respect to $\rho(z), \eta_{1}(z)$, and $\eta_{2}(z)$, i.e.,

$$
\frac{\delta(\Omega / A)}{\delta \rho(z)}=0, \quad \frac{\delta(\Omega / A)}{\delta \eta_{1}(z)}=0, \text { and } \frac{\delta(\Omega / A)}{\delta \eta_{2}(z)}=0,
$$

three coupled integral equations,

$$
\begin{aligned}
\frac{\mu}{k_{B} T}= & \frac{\mu_{h}(\rho(z))}{k_{B} T}+\int d \omega f(z, \omega) \ln [4 \pi f(z, \omega)] \\
& -\frac{\mu_{0} E}{k_{B} T} \eta_{1}(z)+\frac{1}{k_{B} T} \int_{-\infty}^{\infty} d z^{\prime} \phi_{\mathrm{eff}}(\bar{z}) \rho\left(z^{\prime}\right), \\
\alpha_{1}(z)= & \frac{\mu_{0} E}{k_{B} T}-\frac{1}{k_{B} T} \int_{-\infty}^{\infty} d z^{\prime} \rho\left(z^{\prime}\right) \eta_{1}\left(z^{\prime}\right) \phi_{3}(\bar{z}),
\end{aligned}
$$

$$
\alpha_{2}(z)=-\frac{1}{k_{B} T} \int_{-\infty}^{\infty} d z^{\prime} \rho\left(z^{\prime}\right)\left(\eta_{2}\left(z^{\prime}\right) \phi_{1}(\bar{z})+\phi_{2}(\bar{z})\right)
$$

are yielded. This set of integral equations is analogous to that obtained by Teixeira and Telo da Gama, ${ }^{17}$ except the terms containing the electric field in Eqs. (14) and (15).

We will consider only the case of electric field which satisfies the condition

$$
|\alpha(z, \phi)| \ll 1
$$

This condition can be further expressed in terms of $\eta_{1}(z)$ and $\eta_{2}(z)$ by expanding the exponential in Eq. (8) and retaining only the first two nontrivial terms. Then, from Eq. (12), we obtain

$$
\eta_{1}(z)=\alpha_{1}(z) / 3 \text { and } \eta_{2}(z)=\alpha_{2}(z) / 5 .
$$

Combining Eqs. (9) and (17) with (18), it is easy to write the condition (17) in the form

$$
\left|3 \eta_{1}(z) P_{1}(\cos \theta)+5 \eta_{2}(z) P_{2}(\cos \theta)\right| \leqslant 1 .
$$

As a result, the three integral equations (14)-(16) take the new form

$$
\begin{aligned}
\frac{\mu}{k_{B} T}= & \frac{\mu_{h}(\rho(z))}{k_{B} T}+\frac{3}{2} \eta_{1}^{2}(z)+\frac{5}{2} \eta_{2}^{2}(z)-\frac{\mu_{0} E}{k_{B} T} \eta_{1}(z) \\
& +\frac{1}{k_{B} T} \int_{-\infty}^{\infty} d z^{\prime} \phi_{\mathrm{eff}}(\bar{z}) \rho\left(z^{\prime}\right), \\
\eta_{1}(z)= & \frac{\mu_{0} E}{3 k_{B} T}-\frac{1}{3 k_{B} T} \int_{-\infty}^{\infty} d z^{\prime} \rho\left(z^{\prime}\right) \eta_{1}\left(z^{\prime}\right) \phi_{3}(\bar{z}),
\end{aligned}
$$

and

$$
\eta_{2}(z)=-\frac{1}{5 k_{B} T} \int_{-\infty}^{\infty} d z^{\prime} \rho\left(z^{\prime}\right)\left(\eta_{2}\left(z^{\prime}\right) \phi_{1}(\bar{z})+\phi_{2}(\bar{z})\right) .
$$

\section{THE BULK PROPERTIES AND PHASE COEXISTENCE}

In the bulk phase the density $\rho$ and orientation order parameters $\eta_{1}$ and $\eta_{2}$ are uniform constants and independent of the variable $z$. In addition, due to the vanishing of the integral $\int_{-\infty}^{\infty} d z \phi_{2}(\bar{z})$ in Eq. (22),

$$
\eta_{2}=0
$$

Therefore, Eqs. (20)-(22) are reduced to two equations,

$$
\frac{\mu}{k_{B} T}=\frac{\mu_{h}(\rho)}{k_{B} T}+\frac{3}{2} \eta_{1}^{2}-\frac{\mu_{0} E}{k_{B} T} \eta_{1}-\Psi \rho+\frac{8}{3 k_{B} T} \pi \mu_{0}^{2} \eta_{1}^{2} \rho,
$$

where

$$
\begin{aligned}
\Psi= & \left(16 \epsilon_{\mathrm{LJ}} d^{3} / 3 k_{B} T\right) \pi\left(1+\frac{2}{3}\left(\epsilon_{\mathrm{LJ}} / k_{B} T\right)\right. \\
& \left.+\frac{1}{12}\left(\mu_{0}^{4} / k_{B} T d^{6} \epsilon_{\mathrm{LJ}}\right)\right),
\end{aligned}
$$

and

$$
\eta_{1}=\frac{\mu_{0} E}{3 k_{B} T} /\left(1+\frac{8 \pi \mu_{0}^{2}}{9 k_{B} T} \rho\right) .
$$


With account of Eqs. (23)-(25) and (10) the grand canonical potential per unit of volume becomes

$$
\frac{\Omega}{V}=-p_{h}(\rho)+\frac{1}{2} \Psi \rho^{2}-\frac{4}{3 k_{B} T} \pi \mu_{0}^{2} \eta_{1}^{2} \rho^{2},
$$

where $p_{h}=\rho \mu_{h}-f_{h}$ is the hard-sphere pressure. Here, the expressions for $\mu$ [Eq. (24)] and $\Omega$ [Eq. (26)] contain some additional terms involving $\eta_{1}$ because of the presence of the uniform electric field. A similar expression for $\Omega$ was also derived by Woodward and Nordholm ${ }^{35}$ for the dipolar hardsphere fluid, where the first two terms of $\Psi$ are absent because $\epsilon_{\mathrm{LJ}}=0$. Retaining only the terms in the order of $\eta_{1}^{2}$ their expression for $\Omega$ is the same as Eq. (26).

The vapor-liquid phase coexistence under the applied electric field can be determined from solving the phaseequilibrium equations

$$
\mu\left(\rho_{l}\right)=\mu\left(\rho_{v}\right)=\mu,
$$

and

$$
\frac{\Omega\left(\rho_{l}\right)}{V}=\frac{\Omega\left(\rho_{v}\right)}{V}
$$

where $\rho_{l}$ and $\rho_{v}$ are the coexisting liquid and vapor density. Hereafter the thermodynamic variables will be given in the reduced units, $\eta=(\pi / 6) \rho d^{3}, \quad T^{*}=k_{B} T / \epsilon_{\mathrm{LJ}}, \quad E^{*}$ $=E\left(d^{3} / \epsilon_{\mathrm{LJ}}\right)^{1 / 2}$, and $\mu_{0}^{*}=\mu_{0} /\left(d^{3} \epsilon_{\mathrm{LJ}}\right)^{1 / 2}$. In this study, the reduced dipole moment $\mu_{0}^{*}$ is chosen to be 1 .

In Fig. 1, the density-temperature phase diagrams for different values of the electric field are shown. Reference 17 has reported that increasing the dipole moment of molecules will lead to a higher critical temperature $T_{c}^{*}$, i.e., an up-shift of the phase-coexistence curve. Concomitantly, the coexisting vapor density becomes smaller whereas the coexisting liquid density becomes larger at fixed reduced temperature. In contrast, applying a uniform electric field gives the opposite trend as shown in Fig. 1. The stronger the applied field, the lower the critical temperature is (see Table I). The same phase-coexistence behavior in the electric field was found for the slab-shaped system as well as for the spherical shaped system with surrounding dielectric constant $\epsilon^{\prime}=1$ by Woodward and Nordholm. ${ }^{35}$ Kiyohara et al. confirmed the latter case using the Gibbs-ensemble Monte Carlo simulation method. ${ }^{34}$ The opposite behavior of $T_{c}$, that is, $T_{c}$ increases with the applied field, was also found in the needle-shaped system $^{36}$ and the spherical shaped system when $\epsilon^{\prime}$ is equal to the dielectric constant of the system ${ }^{34}$ or $\epsilon^{\prime}=\infty .{ }^{32-34}$ In summary, the phase-coexistence behavior of the dipolar system is sensitive to the shape of the system and to the dielectric constant of the surrounding media in the presence of an external electric field.

In Fig. 2, we plot the phase-coexistence curves of the orientation order parameter $\eta_{1}$. In the absence of the electric field, $\eta_{1}=0$. With increasing of the field, $\eta_{1}$ increases too. We found $\eta_{1}$ is more sensitive to the strength of the electric field, compared to $\rho$. In contrast to the behavior of $\rho$, larger values of $\eta_{1}$ correspond to the coexisting vapor phase whereas smaller values $\eta_{1}$ correspond to the liquid phase. Physically, $\eta_{1}$ represents the average cosine of the angle between the direction of the electric field and direction of the
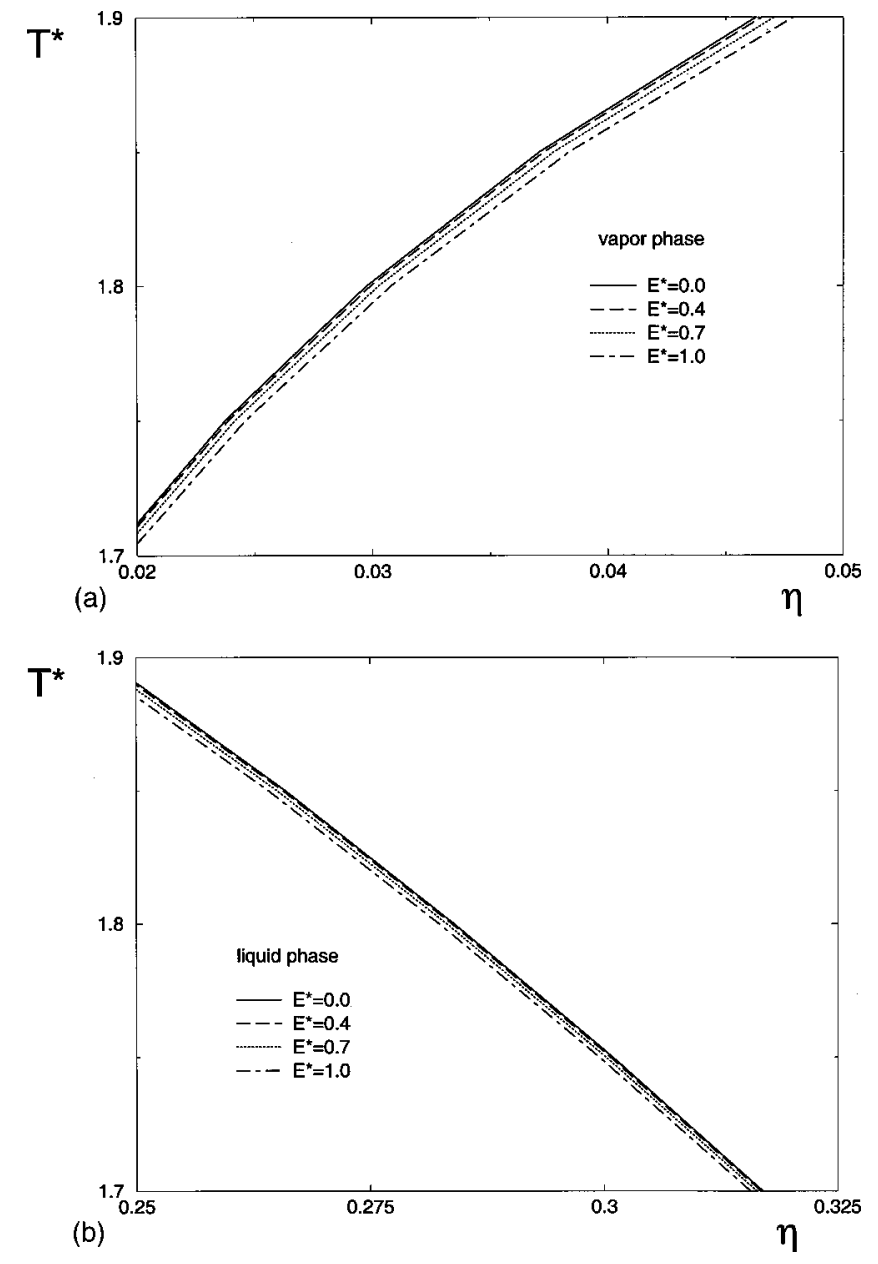

FIG. 1. Vapor-liquid coexistence curves for different values of electric field $E^{*}$. (a) The vapor branch and (b) the liquid branch.

dipole moments. The reason that $\eta_{1}$ is larger in the vapor is because relatively more molecules in the vapor tend to orient along the direction of the electric field.

Since Eqs. (20)-(22) are obtained under condition (19), we need to verify whether the obtained solutions are consistent with this condition. In the bulk phase $\eta_{2}=0$, thus, we only need to examine $\eta_{1}$ in the vapor phase. Because $\eta_{1 l}$ $<\eta_{1 v}$, if condition (19) is satisfied in the vapor phase it should also work for the liquid phase. Using the fact that in the vapor phase the second term in the denominator in Eq. (25) is negligible, Eq. (19) becomes

$$
\mu_{0} E / k_{B} T \ll 1 \text {. }
$$

This condition is found to be satisfied for all values of the electric field and temperature considered in this work.

TABLE I. Critical temperature $T_{c}^{*}$, density $\eta_{c}$, order parameter $\eta_{1 c}$, and the dielectric constant $\epsilon_{c}$ as a function of the field $E^{*}$.

\begin{tabular}{ccccc}
\hline \hline$E^{*}$ & $T_{c}^{*}$ & $\eta_{c}$ & $\eta_{1 c}$ & $\epsilon_{c}$ \\
\hline 0.0 & 2.0590 & 0.1304 & 0.0000 & 1.6098 \\
0.4 & 2.0580 & 0.1305 & 0.0484 & 1.6106 \\
0.7 & 2.0558 & 0.1306 & 0.0848 & 1.6121 \\
1.0 & 2.0525 & 0.1309 & 0.1212 & 1.6145 \\
\hline \hline
\end{tabular}




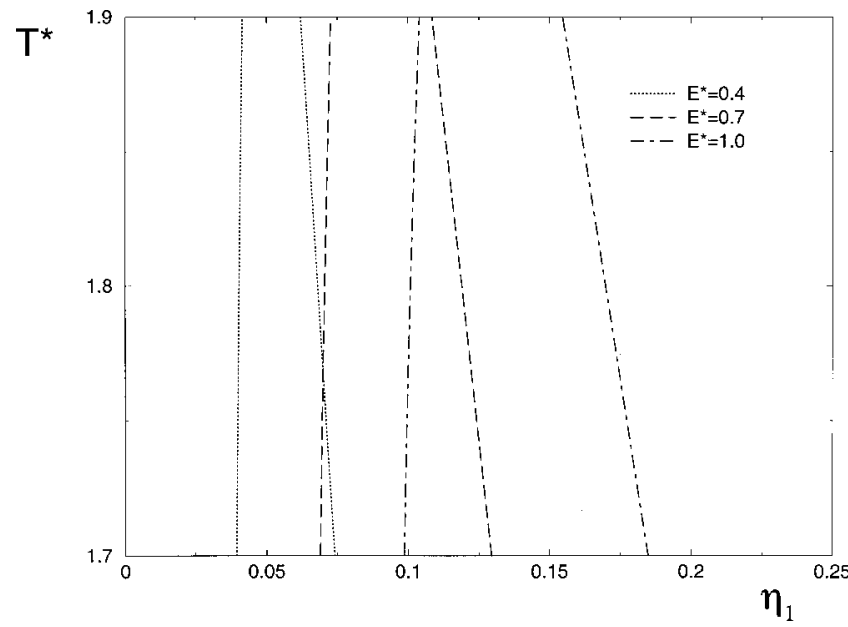

FIG. 2. The orientation order parameter $\eta_{1}$ at phase coexistence for different values of electric field $E^{*}$. At fixed $E^{*}$ the branch with the larger values of $\eta_{1}$ corresponds to the vapor phase and the branch with the smaller values of $\eta_{1}$ corresponds to the liquid phase.

We also determined the dielectric constants of coexisting vapor and liquid phases. The dielectric constant $\epsilon$ of a bulk phase is defined via the equation

$$
\epsilon=1+4 \pi \frac{P}{E_{M}},
$$

where $E_{M}$ is the so-called Maxwell field and $P$ is the polarization,

$$
P=\mu_{0} \eta_{1} \rho .
$$

The Maxwell field depends on the shape of the system. ${ }^{35}$ Specifically, for the slab-shaped system the Maxwell field is given by ${ }^{35,40}$

$$
E_{M}=E-4 \pi P .
$$

Combining Eq. (29) with Eq. (31) yields

$$
\epsilon=\frac{1}{1-\frac{4 \pi P}{E}} .
$$

Furthermore, substituting Eqs. (25) and (30) in Eq. (32) we arrive at the well-known Clausius-Mosotti formula

$$
\frac{\epsilon-1}{\epsilon+2}=\frac{4 \pi}{9} \frac{\mu_{0}^{2}}{k_{B} T} \rho,
$$

a result from the mean-field approximation for the slabshaped system. In Ref. 41 the same formula for dielectric permittivity is also obtained by use of the mean-field approximation but for a spherical shaped system.

The dielectric constants of the coexisting vapor and liquid phase are depicted in Fig. 3. One can see that for the given temperatures, there is no "Clausius-Mosotti catastrophe," i.e., $\epsilon$ remains finite. At a fixed temperature the dielectric constant decreases in the liquid phase but increases in the vapor phase with increasing strength of the electric field. The dependence of the dielectric constant of the coexisting phases on the electric field can be explained as follows. In an open system the dielectric constant is a function of the
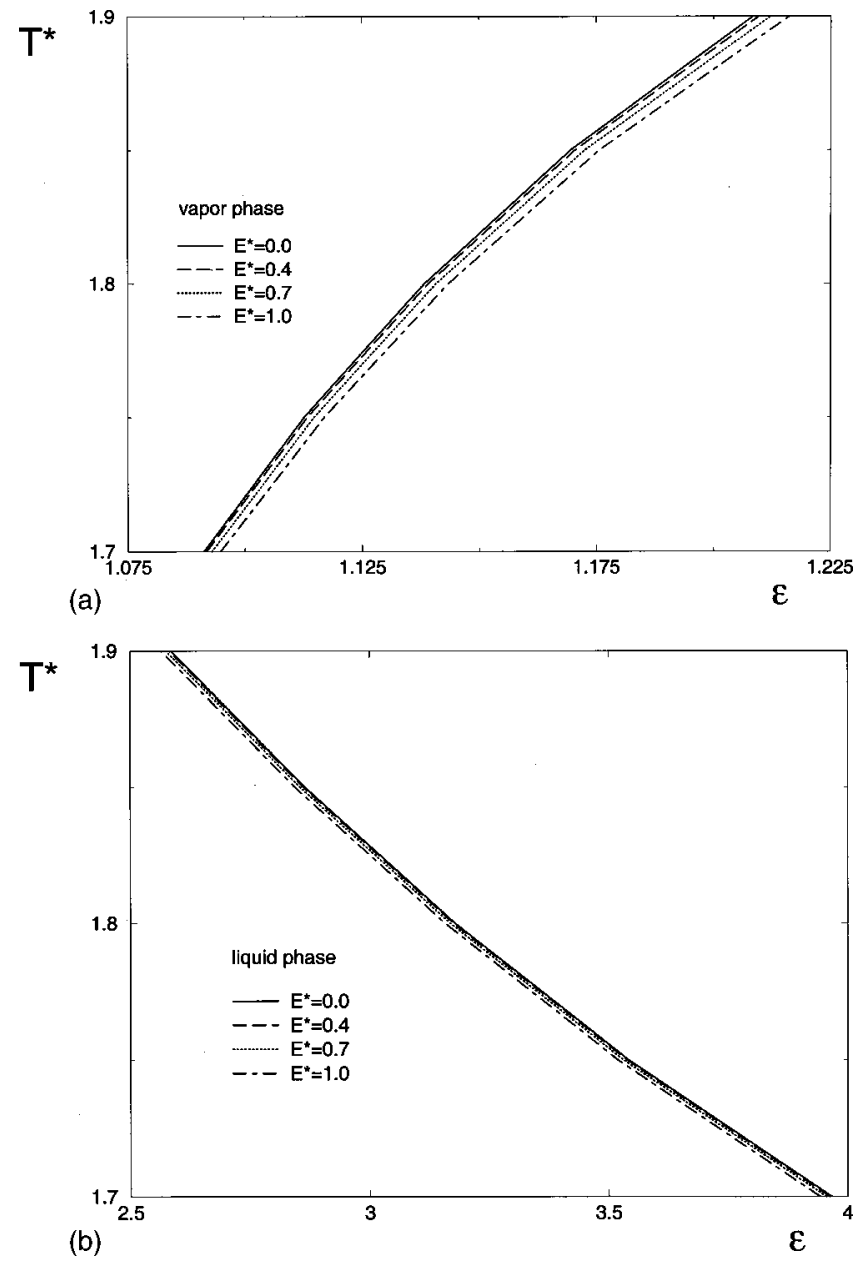

FIG. 3. The dielectric constant $\epsilon$ at phase coexistence for different values of electric field $E^{*}$. (a) The vapor branch and (b) the liquid branch.

chemical potential and temperature, i.e., $\epsilon=\epsilon(\mu, T)$. When $T$ is fixed, only $\mu$ can be altered by changing the electric field and so does $\epsilon$.

\section{INTERFACIAL PROPERTIES}

The planar vapor-liquid interfacial density and orientation order-parameter profiles can be obtained by solving the integral equations (20)-(22) numerically using an iteration method. Here we used a step function as an initial input for $\rho(z)$ and $\eta_{1}(z)$. The step function has the bulk liquid value at $z=-\infty$ and vapor value at $z=+\infty$. The initial guess for $\eta_{2}(z)$ is just zero. Note that due to the linear nature of Eqs. (21) and (22) there exists a limiting condition on the convergence of the iteration. The Fredholm theorem for the linear integral equations ${ }^{42}$ states that convergence of iteration is possible only if the norm of the kernel of the integral operator is less than 1. For Eq. (21), the Fredholm theorem requires the existence of a constant $C$ such that

$$
\frac{1}{3 k_{B} T} \int_{-\infty}^{\infty} d z^{\prime}\left|\rho\left(z^{\prime}\right) \phi_{3}(\bar{z})\right|<C<1
$$

for all values of $z$. This limiting condition could be broken in the liquid region where density is high. A simple estimation 
of the integral in Eq. (34) can be made with replacing $\rho(z)$ by the bulk value $\rho_{l}$ so that Eq. (34) is simplified to

$$
\frac{8 \pi}{9} \frac{\mu_{0}^{2}}{k_{B} T} \rho_{l}<C<1 .
$$

Equation (35) gives the upper bound to $\rho_{l}$ for given temperature and dipole moment.

Another limiting condition is Eq. (19). We note that for the magnitude of electric field considered here the inequality

$$
\max _{z} \eta_{1}(z)=\eta_{1 v} \gg\left|\max _{z} \eta_{2}(z)\right|
$$

always holds. Substitution of Eq. (36) into condition (19) yields Eq. (28) for all the dipole moments and temperatures considered here.

Figure 4(a) shows the reduced density profiles $\eta(z)$ at various reduced temperatures and fixed electric field $E^{*}$ $=1$. Results for orientation order parameters $\eta_{1}(z)$ and $\eta_{2}(z)$ are presented in Figs. 4(b) and 4(c). Figures 5(a) and 5(b) show the orientation order parameters $\eta_{1}$ and $\eta_{2}$ profiles, respectively, at different values of the electric field and temperature $T^{*}=1.8$. The obtained $\eta_{1}$ with $\eta_{2}$ satisfy the inequality (36). As mentioned above, $\eta_{1}(z)$ is more sensitive to the electric field and it increases at any $z$ with increasing electric field. The physical interpretation of the behavior of $\eta_{2}(z)$ at the interfacial region has been given in several papers. ${ }^{17,22}$ On the liquid side, $\eta_{2}(z)<0$ because the molecular dipoles prefer to lie in parallel with the interface while on the vapor side, $\eta_{2}(z)>0$ because the dipoles prefer to be normal to the interface. In the electric field the obtained positive values of $\eta_{1}(z)$ show that the dipolar direction along the field is more favorable.

At the vapor-liquid interface, the dielectric constant becomes a function of $z$. However, since the induction of the Maxwell field, $D_{M}$, is equal to $E$ anywhere in the interface Eq. (31) is still valid even though $E_{M}$ depends on $z$ there. The dielectric constant profile $\epsilon(z)$ can still be calculated using Eq. (32) in which the local polarization profile

$$
P(z)=\mu_{0} \eta_{1}(z) \rho(z) .
$$

In Fig. 6 we plot $\epsilon(z)$ for different temperatures.

We also examined the influence of the external electric field on the surface tension. Note that when the planar vapor-liquid interface is in the presence of an electric field the definition of the surface tension is no longer unique. One can define both thermodynamical surface tension $\sigma$ and mechanical surface tension $\gamma$ at any dividing surfaces. The former is more widely used because $\sigma$, defined as the excess of the grand canonical potential per unit of area

$$
\sigma=\left(\Omega-\Omega^{\mathrm{bulk}}\right) / A,
$$

is independent of the choice of the dividing surface. Figure 7(a) shows the dependence of $\sigma$ on the temperature for different values of the electric field. It is known ${ }^{17,20}$ that $\sigma$ increases with increasing value of the dipole moment at fixed temperature. From Fig. 7(a) one can see that the field effect is opposite, i.e., $\sigma$ decreases with increasing strength of the electric field.

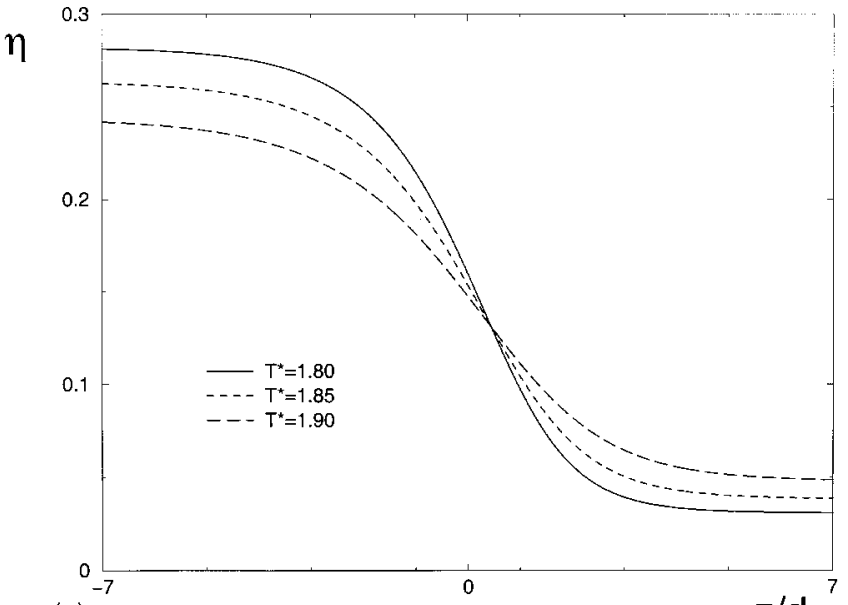

(a)

$\mathrm{z} / \mathrm{d}$
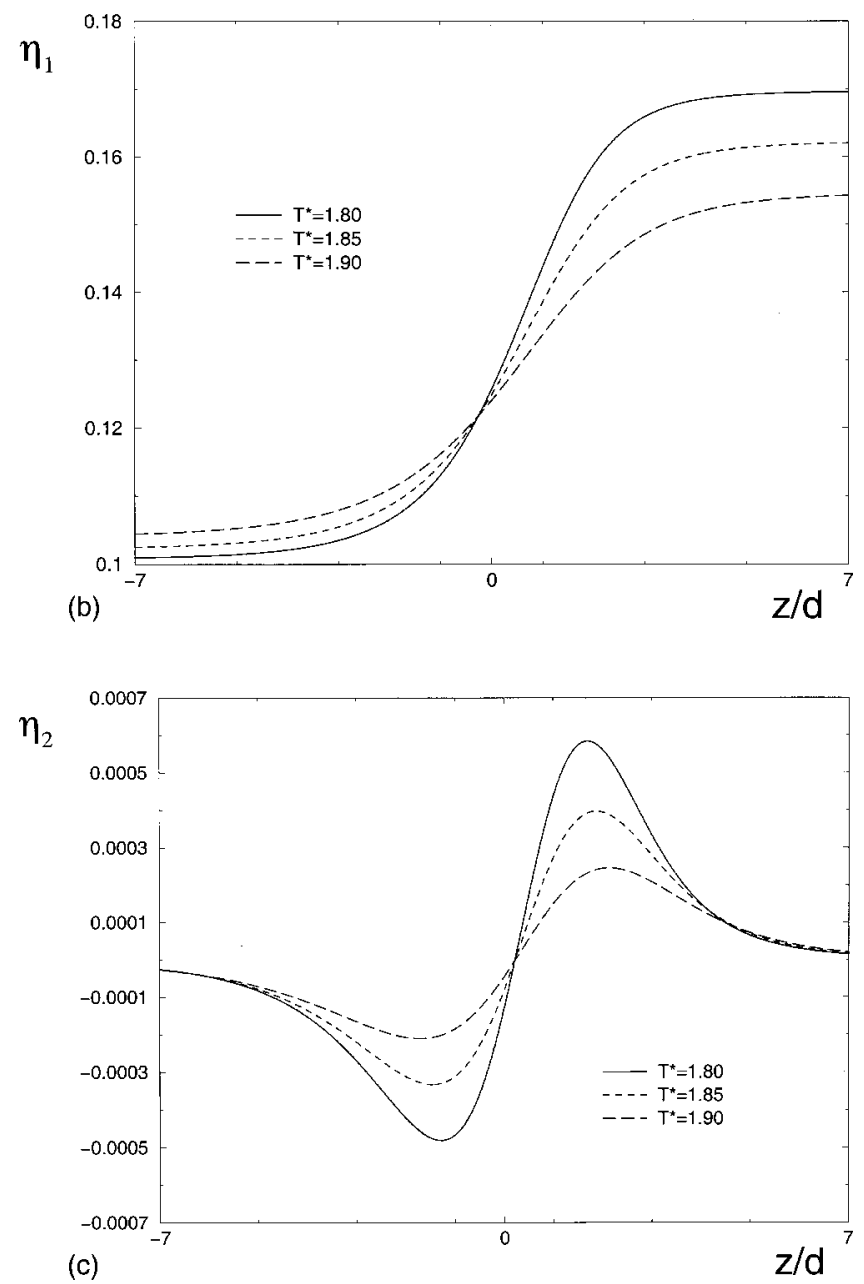

FIG. 4. (a) $\eta(z)$, (b) $\eta_{1}(z)$, and (c) $\eta_{2}(z)$ in the interfacial region for different values of temperature $T^{*}$. The electric field $E^{*}=1$.

The mechanical surface tension $\gamma$ can be defined based on the thermodynamical surface tension $\sigma$. Starting with the grand canonical potential in the form ${ }^{24}$

$$
\Omega=-A \int_{-\infty}^{+\infty} p_{T}(z) d z-A \int_{-\infty}^{+\infty} P(z) E d z,
$$

where $p_{T}$ is the tangential component of the pressure tensor in the external electric field, Eq. (38) can be rewritten as 


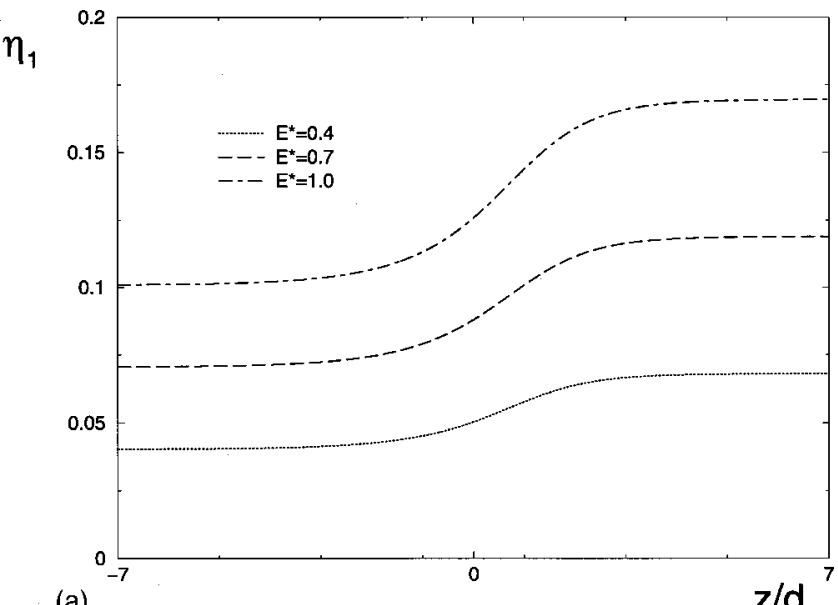

(a)

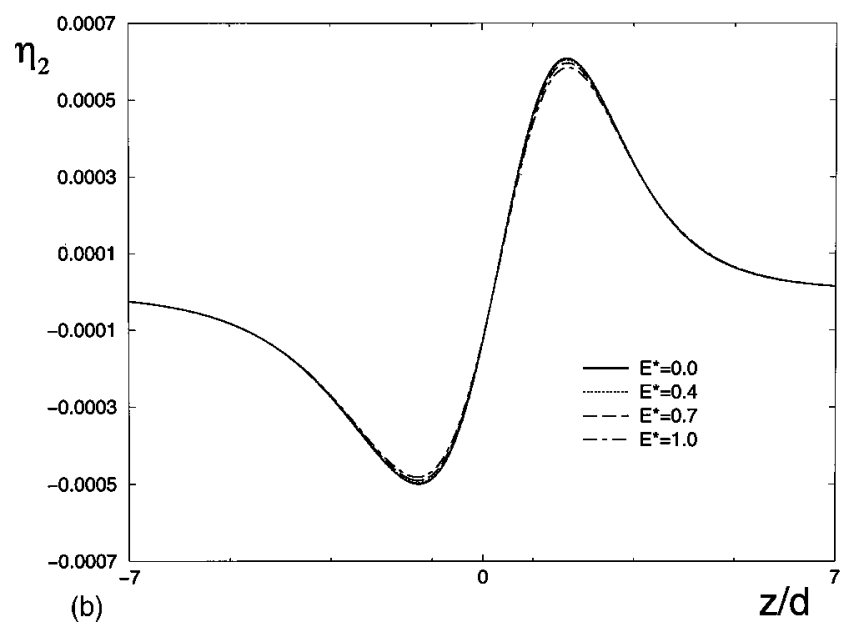

FIG. 5. (a) $\eta_{1}(z)$ and (b) $\eta_{2}(z)$ in the interfacial region for different values of electric field $E^{*}$. The temperature $T^{*}=1.8$.

$$
\sigma=\gamma-\bar{P} E,
$$

where $\gamma$ is the mechanical surface tension

$$
\gamma=-\int_{-\infty}^{Z}\left(p_{T}(z)-p_{T}^{l}\right) d z-\int_{Z}^{+\infty}\left(p_{T}(z)-p_{T}^{v}\right) d z,
$$

and $\bar{P}$ is the excess polarization

$$
\bar{P}=\int_{-\infty}^{Z}\left(P(z)-P^{l}\right) d z+\int_{Z}^{+\infty}\left(P(z)-P^{v}\right) d z,
$$

and $Z$ is the position of the dividing surface. Because of the inequality

$$
P^{v} \leqslant P(z) \leqslant P^{l},
$$

the first integral in Eq. (42) is less than zero while the second integral is greater than zero for any $Z$. As a result, one can choose a dividing surface such that $\bar{P}=0$. As such, the thermodynamic and mechanical surface tensions become identical $(\sigma=\gamma)$.

In Fig. 7(b) we show the results of $\gamma$ at the equimolar dividing surface $Z_{e}$. The latter is defined from the equation

$$
\int_{-\infty}^{Z_{e}}\left(\rho(z)-\rho_{l}\right) d z+\int_{Z_{e}}^{+\infty}\left(\rho(z)-\rho_{v}\right) d z=0,
$$

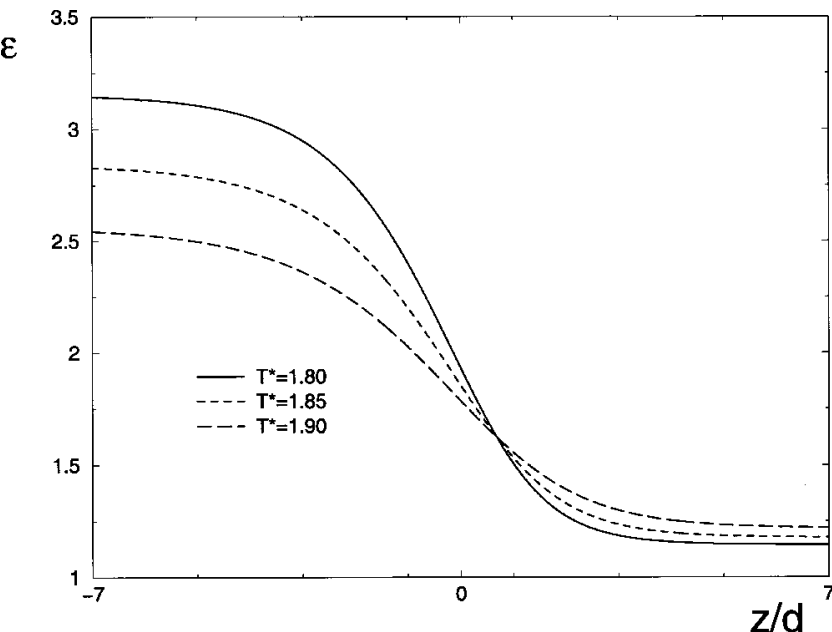

FIG. 6. The dielectric constant profile $\epsilon(z)$ in the interfacial region for different values of temperature. The electric field $E^{*}=1$.

at which the excess polarization is positive. In contrast to the behavior of $\sigma$ in the field, $\gamma$ tends to increase with increasing electric field. We note that this result can only be used for the slab-shaped system. Generally, the behavior of the surface tension should depend on the shape of the system in the electric field.

As aforementioned, the thermodynamical surface tension $\sigma$ has a theoretical advantage in that $\sigma$ is independent of the choice of the dividing surface. In practice, however, only the mechanical surface tension $(\gamma)$ can be measured directly either from experiments ${ }^{43}$ or from computer simulations. ${ }^{44}$ In the absence of an external electric field, the measured surface tension $\gamma$ is essentially the same as the thermodynamic surface tension $\sigma$, which is independent of the location of the dividing surface. In the presence of the field, the measured surface tension $\gamma$ will in principle depend on the location of the dividing surface. However, as shown in Fig. 7(b), the effects of the field on $\gamma$ is generally small (a few percent change in $\gamma$ ) when $\mu_{0} E / k_{B} T \ll 1$. Therefore, high-resolution surface-tension measurements are needed to detect this change in $\gamma$. Thus far, we are not aware of any experimental measurements of the field dependence of surface tension.

How strong an electric field $E$ can be used without violating the condition $\mu_{0} E / k_{B} T \ll 1$ ? We used the Stockmayer model (which is very similar to the model used in this work) of methanol as an example to estimate the upper limit of $E$. The Stockmayer parameters of methanol have been derived by van Leeuwen: ${ }^{45} \epsilon / k_{B}=359.0 \mathrm{~K}, \sigma=3.803 \AA$, and $\mu_{0}^{*}$ $=1.036$. Thus, from the condition $\mu_{0} E / k_{B} T \ll 1$ (or $\left.\mu_{0}^{*} E^{*} / T^{*} \ll 1\right)$ we find $E$ should be much less than 4.5 $\times 10^{8} \mathrm{~V} / \mathrm{m}$ provided $T^{*}=1$. From this estimation we suspect that a relatively strong field, e.g., $E \sim 10^{8} \mathrm{~V} / \mathrm{m}$, may be needed to detect the shift in $\gamma$. A higher field may cause even a larger shift in $\gamma$, however, a quantitative prediction for this shift is beyond the limitation of the present theory (which works only for weakly dipolar systems). To see the field effects on the thermodynamic surface tension $\sigma$, on the other hand, one should use Eq. (40). Thus, one needs to measure the excess polarization ${ }^{46-49}$ also. The latter is responsible to the predicted reduction of $\sigma$ as the field strength increases. 

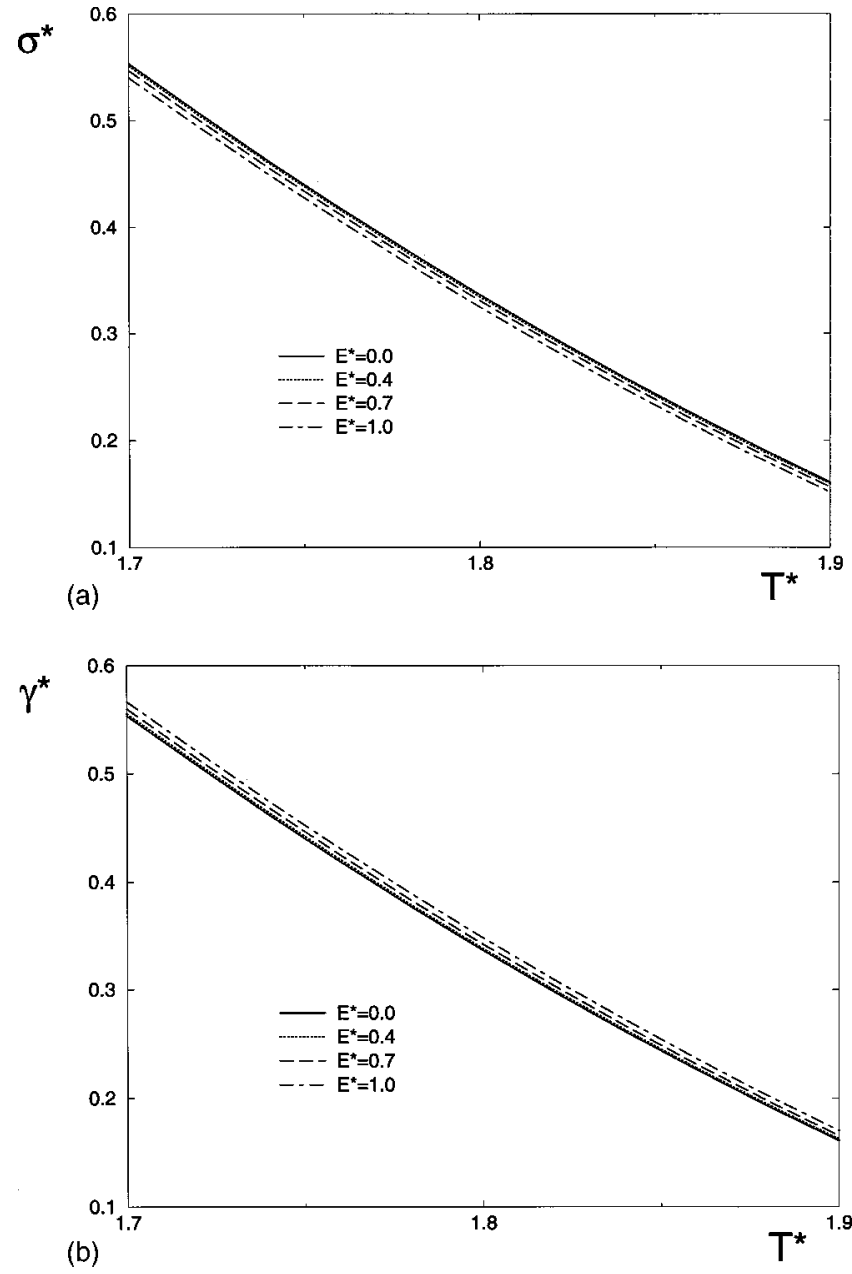

FIG. 7. Temperature dependence of (a) reduced thermodynamical surface tension $\sigma^{*}=\sigma d^{2} / \epsilon_{\mathrm{LJ}}$ and (b) reduced mechanical surface tension $\gamma^{*}$ $=\gamma d^{2} / \epsilon_{\mathrm{LJ}}$ for different values of electric field $E^{*}$.

To close, we would like to address the question about the influence of the direction of the electric field on the interfacial properties of the fluid. The linearization of the set of Eqs. (14)-(16) in the case of the electric field satisfying condition (17) results in the fact that Eqs. (20)-(22) are symmetric under reversing the direction of the field. This is because only $\eta_{1}(z)$ changes sign, whereas $\rho(z)$ and $\eta_{2}(z)$ do not change upon reversing the field direction. With this linearization the grand canonical potential [Eqs. (10) and (26)] does not depend on the direction of the applied field because only $E^{2}$ terms show up in the equations. As a result, the direction of the field will not affect the surface tension.

\section{CONCLUSIONS}

We have extended the MMF density-functional theory to the case of weakly dipolar fluids $\left(\mu_{0}^{*} \leqslant 1\right)$ in the presence of a uniform electric field. We find that the applied field tends to increase the equilibrium vapor density but decrease the liquid density for the slab system. Similar behavior has also been found in a different slab system ${ }^{35}$ and in the spherical system with $\epsilon^{\prime}=1 .^{35,34}$ The influence of the electric field on the density, orientation order-parameter and dielectric constant profiles is also examined. We have shown that for the slab system at fixed temperature, increasing the applied field tends to reduce the thermodynamical surface tension but enhance the mechanical surface tension at the equimolar dividing surface. To detect this opposite trend in the two forms of surface tension, a relatively strong field, e.g., $E \sim 10^{8} \mathrm{~V} / \mathrm{m}$, may be needed. Measurement of excess polarization at different dividing surfaces is also required to determine the thermodynamic surface tension.

\section{ACKNOWLEDGMENTS}

We are grateful to Professor A. Shchekin, Professor V. Kuz'min, and Dr. K. J. Oh for useful discussions and comments. This work is supported by the National Science Foundation.

${ }^{1}$ J. J. Thomson, Conduction of Electricity Through Gases (Cambridge University Press, Cambridge, 1906).

${ }^{2}$ M. Volmer, Kinetik der phasenbildung (Steinkopff, Dresden, 1939).

${ }^{3}$ A. I. Rusanov and F. M. Kuni, J. Colloid Interface Sci. 100, 264 (1984).

${ }^{4}$ H. Rabeony and P. Mirabel, J. Phys. Chem. 91, 1815 (1987).

${ }^{5}$ M. Adachi, K. Okumura, and J. H. Seinfeld, J. Aerosol Sci. 23, 327 (1992).

${ }^{6}$ F. He and P. K. Hopke, J. Chem. Phys. 99, 9972 (1993).

${ }^{7}$ J. L. Katz, J. A. Fisk, and V. M. Chakarov, J. Chem. Phys. 101, 2309 (1994).

${ }^{8}$ D. Kane, G. M. Daly, and M. S. El-Shall, J. Phys. Chem. 99, 7867 (1995).

${ }^{9}$ I. Kusaka, Z.-G. Wang, and J. H. Seinfeld, J. Chem. Phys. 102, 913 (1995).

${ }^{10}$ V. Talanquer and D. W. Oxtoby, J. Chem. Phys. 103, 3686 (1995).

${ }^{11}$ D. Kashchiev, Philos. Mag. 25, 459 (1972).

${ }^{12}$ J. O. Isard, Philos. Mag. 35, 817 (1977).

${ }^{13}$ K. J. Cheng, Phys. Lett. 106, 403 (1984).

${ }^{14}$ K. J. Oh, G. T. Gao, and X. C. Zeng, J. Chem. Phys. 108, 4683 (1998)

${ }^{15}$ G. T. Gao, K. J. Oh, and X. C. Zeng, J. Chem. Phys. 110, 2533 (1999).

${ }^{16}$ V. B. Warshavsky and A. K. Shchekin, Colloids Surf., A 148, 283 (1999).

${ }^{17}$ P. I. Teixeira and M. M. Telo da Gama, J. Phys.: Condens. Matter 3, 111 (1991).

${ }^{18}$ P. I. Teixeira, B. S. Almeida, M. M. Telo da Gama et al., J. Phys. Chem. 96, 8488 (1992).

${ }^{19}$ V. Talanquer and D. W. Oxtoby, J. Chem. Phys. 99, 4670 (1993).

${ }^{20}$ P. Frodl and S. Dietrich, Phys. Rev. A 45, 7330 (1992).

${ }^{21}$ P. Frodl and S. Dietrich, Phys. Rev. E 48, 3741 (1993).

${ }^{22}$ M. Kasch and F. Forstmann, J. Chem. Phys. 99, 3037 (1993).

${ }^{23}$ S. Iatzevich and F. Forstmann, Mol. Phys. 98, 1309 (2000).

${ }^{24}$ E. N. Brodskaya and A. I. Rusanov, Colloid J. USSR 45, 558 (1983).

${ }^{25}$ D. G. Hall and B. A. Pethica, Proc. R. Soc. London, Ser. A 364, 457 (1978).

${ }^{26}$ A. I. Rusanov and V. L. Kuz'min, Colloid J. USSR 39, 388 (1977).

${ }^{27}$ L. Liggeri, A. Sanfeld, and A. Steinchen, Physica A 206, 299 (1994).

${ }^{28}$ C. N. Patra and S. K. Ghosh, J. Chem. Phys. 106, 2752 (1997).

${ }^{29}$ D. Das, S. Separati, and A. Chandra, J. Chem. Phys. 110, 8129 (1999).

${ }^{30}$ H. G. Petersen, S. W. de Leeuw, and J. W. Perram, Mol. Phys. 66, 637 (1989).

${ }^{31}$ P. G. Kusalik, Mol. Phys. 81, 199 (1994).

${ }^{32}$ M. J. Stevens and G. S. Grest, Phys. Rev. E 51, 5976 (1995).

${ }^{33}$ D. Boda, J. Winkelmann, J. Liszi, and I. Szalai, Mol. Phys. 87, 601 (1996).

${ }^{34}$ K. Kiyohara, K. J. Oh, X. C. Zeng, and K. Ohta, Mol. Simul. 23, 95 (1999).

${ }^{35}$ C. E. Woodward and S. Nordholm, J. Phys. Chem. 92, 501 (1988).

${ }^{36}$ B. Groh and S. Dietrich, Phys. Rev. E 53, 2509 (1996).

${ }^{37}$ G. Gray and K. E. Gubbins, The Theory of Molecular Fluids (Clarendon, Oxford, 1984)

${ }^{38}$ M. E. Rose, Elementary Theory of Angular Momentum (Wiley, New York, 1957).

${ }^{39}$ N. E. Carnahan and K. E. Starling, J. Chem. Phys. 51, 635 (1969).

${ }^{40}$ J. S. Høye and G. Stell, J. Chem. Phys. 72, 1597 (1980). 
${ }^{41}$ J. S. Høye and G. Stell, J. Chem. Phys. 61, 562 (1974).

${ }^{42}$ S. L. Sobolev, Uravneniya Matematicheskoy Fiziki (Moscow, Nauka, 1992).

${ }^{43}$ A. I. Rusanov and V. A. Prokhorov, Interfacial Tensiometry (Amsterdam, New York, 1996).

${ }^{44}$ M. J. Haye and C. Bruin, J. Chem. Phys. 100, 556 (1994).

${ }^{45}$ M. E. van Leeuwen, Fluid Phase Equilibria 99, 1 (1994).
${ }^{46}$ N. N. Kochurov, B. A. Noskov, and A. I. Rusanov, Dokl. Akad. Nauk SSSR 227, 1386 (1976).

${ }^{47}$ R. Gomer and G. Tryson, J. Chem. Phys. 66, 4413 (1977).

${ }^{48}$ J. R. Farrell and P. McTigue, J. Electroanal. Chem. Interfacial Electrochem. 139, 37 (1982).

${ }^{49}$ M. A. Wilson, A. Pohorille, and L. R. Pratt, J. Chem. Phys. 88, 3281 (1988). 